\title{
Nuclear Factor Erythroid 2-related Factor 2 Knockout Suppresses the Development of Aggressive Colorectal Cancer Formation Induced by Azoxymethane/Dextran Sulfate Sodium-Treatment in Female Mice
}

\author{
Chin-Hee Song', Nayoung Kim,2, Ryoung Hee Nam, Soo In Choi', Changhee Kang1, Jae Young Jang', \\ Heewon $\mathrm{Nho}^{1}$, Eun Shin ${ }^{3}$, Ha-Na Lee ${ }^{4}$ \\ ${ }^{1}$ Department of Internal Medicine, Seoul National University Bundang Hospital, Seongnam, ${ }^{2}$ Department of Internal \\ Medicine and Liver Research Institute, Seoul National University College of Medicine, Seoul, ${ }^{3}$ Department of Pathology, \\ Hallym University Dongtan Sacred Heart Hospital, Hwaseong, Korea, ${ }^{4}$ Laboratory of Immunology, Division of Biotechnology \\ Review and Research-III, Office of Biotechnology Products, Center for Drug Evaluation and Research, Food and Drug \\ Administration, Silver Spring, MD, USA
}

\begin{abstract}
Colon tumors develop more frequently in male than in female. Nuclear factor erythroid 2-related factor 2 (Nrf2) plays differential roles in the stage of tumorigenesis. The purpose of this study was to investigate the role of Nrf2 on colitis-associated tumorigenesis using Nrf2 knockout (KO) female mice. Azoxymethane (AOM) and dextran sulfate sodium (DSS)-treated wild-type (WT) and Nrf2 $\mathrm{KO}$ female mice were sacrificed at week 2 and 16 after AOM injection. Severity of colitis, tumor incidence, and levels of inflammatory mediators were evaluated in AOM/DSS-treated WT and Nrf2 KO mice. Furthermore, qRT-PCR, Western blot abnalysis, and ELISA were performed in colon tissues. At week 2, AOM/DSS-induced colon tissue damages were significantly greater in Nrf2 KO than in WT mice. At week 16, tumor numbers (> $2 \mathrm{~mm}$ size) were significantly lower in both the proximal and distal colon in Nrf2 $\mathrm{KO}$ compared to WT. The overall incidences of adenoma/cancer of the proximal colon and submucosal invasive cancer of the distal colon were reduced by Nrf2 KO. The mRNA and protein expression levels of NF-кB-related mediators (i.e., iNOS and COX-2) and Nrf2-related antioxidants (i.e., heme oxygenase-1 and glutamate-cysteine ligase catalytic subunit) were significantly lower in the Nrf2 KO than in WT mice. Interestingly, the protein level of 15-hydroxyprostaglandin dehydrogenase (15-PGDH) was higher in AOM/DSS-treated Nrf2 KO than in WT mice. Our results support the oncogenic effect of Nrf2 in the later stage of carcinogenesis and upregulation of tumor suppressor 15-PGDH might contribute to the repression of colitis-associated tumorigenesis in Nrf2 KO female mice.
\end{abstract}

Key Words Colitis-associated carcinogenesis, Nrf2 knockout, AOM/DSS mouse model, Colon cancer, 15-PGDH

\section{INTRODUCTION}

Colorectal cancer (CRC) is the third leading cause of cancer-associated death in both male and female in the United States, with an estimation of 149,500 new cancer cases and 52,980 cancer deaths, in 2021 [1]. In South Korea, male have a higher incidence rate of age-adjusted CRC than female [2]. However, elderly female over 65 years of age show a higher mortality of CRC and a lower 5-year survival rate compared to their age-adjusted male [2]. CRC shows sex differences worldwide in the incidence and the developing site (proximal or distal colon), and underlying mechanisms [3].

Nuclear factor erythroid 2-related factor 2 (Nrf2) is a transcription factor that plays a pivotal role in adaptive cellular defense response to a variety of stimuli such as oxidation, proteotoxic stress, metabolic stress, and inflammation $[4,5]$. In normal cells under non-stress conditions, Nrf2 levels and activity are kept relatively low by its rapid proteasomal degradation which is facilitated by Kelch-like $\mathrm{ECH}$-associated protein 1 (Keap1) [6]. Under stress conditions, however, Nrf2

Received March 7, 2021, Revised March 16, 2021, Accepted March 17, 2021

Correspondence to Nayoung Kim, E-mail: nayoungkim49@empas.com, https://orcid.org/0000-0002-9397-0406

Check for updates 
dissociates from Keap1 and then translocates to the nucleus [4]. Nrf2 activates anti-oxidant enzymes, such as heme oxygenase-1 (HO-1), NAD(P)H: quinone oxidoreductase 1 (NQO1), glutamate-cysteine ligase catalytic subunit (GCLC), and glutamate-cysteine ligase modifier subunit (GCLM), that maintain cellular homeostasis and exhibit anti-inflammatory and anti-tumor activity $[7,8]$. The anti-inflammatory mechanisms of Nrf2 include suppression of pro-inflammatory cytokines such as interleukin (IL)-6, IL-1 $\beta$, and TNF- $\alpha$ by blocking the NF-kB signaling $[9,10]$.

Recently, there have been many reports on the dual roles of Nrf2 in carcinogenesis. Activation of Nrf2 in normal cells prevents them from transformation into cancer cells, whereas activation of Nrf2 in transformed or cancerous cells promotes their growth and survival [11]. Meta-analysis showed that patients with high Nrf2 expression had a lower overall survival rate and disease-free survival compared to those with low Nrf2 expression [12]. In addition, high Nrf2 levels were associated with poor prognosis in CRC patients [13]. Furthermore, overexpression of Nrf2 target genes such as $\mathrm{HO}-1$ and NQO1 has been reported in prostate cancer and CRC, respectively $[14,15]$. Further, Nrf2 overexpression accounts for chemotherapy resistance in many malignancies including gastric and colon cancer $[13,16]$.

Azoxymethane (AOM) and dextran sulfate sodium (DSS) treatments [17-19] are the most widely used protocols for the establishment of animal models of colon carcinogenesis [20-22]. This model well reflects multistep nature of tumor development and progression based on the aberrant crypt foci (ACF)-adenoma-carcinoma sequence with the relevant molecular alterations [21]. Therefore, the Nrf2 KO ( $\mathrm{Nrf}^{-/-}$) AOM/ DSS mouse model could be a useful tool to clarify the role of Nrf2 by sex in colitis and colon tumorigenesis.

We previously reported that $17 \beta$-estradiol $(10 \mathrm{mg} /$ $\mathrm{kg}$ ) reduced the expression of Nrf2 upregulated in AOM/ DSS-treated male ICR mice and suppressed the occurrence of colitis-related CRC to a level similar to that of females [23]. However, the inhibitory effect of $17 \beta$-estradiol $(10 \mathrm{mg} / \mathrm{kg})$ on expression of Nrf2 in C57BL/6 background male mice (wildtype, WT) was not sufficient to inhibit tumorigenesis in the distal colon [24]. In contrast, in the absence of Nrf2 (Nrf2 KO), $17 \beta$-estradiol $(10 \mathrm{mg} / \mathrm{kg})$ strongly inhibited tumorigenesis in the distal colon through an Nrf2-independent estrogen receptor beta (ER $\beta$ )-related signaling pathway [24]. From these observations, we hypothesized that the contribution of Nrf2 to colitis-associated colon tumorigenesis could vary by sex. To test this hypothesis, we investigated the effect of Nrf2 on colitis-associated colon tumorigenesis in AOM/DSS-treated Nrf2 $\mathrm{KO}\left(\mathrm{Nrf}^{-/-}\right)$and WT $\left(\mathrm{Nrr}^{+/+}\right)$female mice.

\section{MATERIALS AND METHODS}

\section{Genotyping and selection of Nrf2 knockout female mice}

Heterozygous Nrf2 KO (Nrf2 ${ }^{+-}$) mice of C57BL6/129SV background generated by the laboratory of Yuet Wai Kan [25] were kindly provided by Prof. Y.-J. Surh of Seoul National University. WT (Nrf2 ${ }^{+/+}$) and homozygous Nrf2 KO (Nrf2 ${ }^{-/-}$) mice were obtained by crossing the Nrf2 heterozygous $\left(\mathrm{Nrf2}^{+/-}\right)$mice (Fig. 1A) as described previously [24]. The mice

A
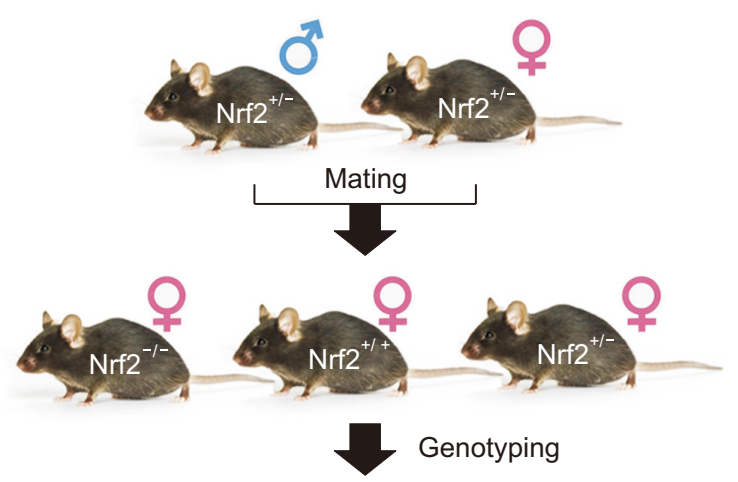

B

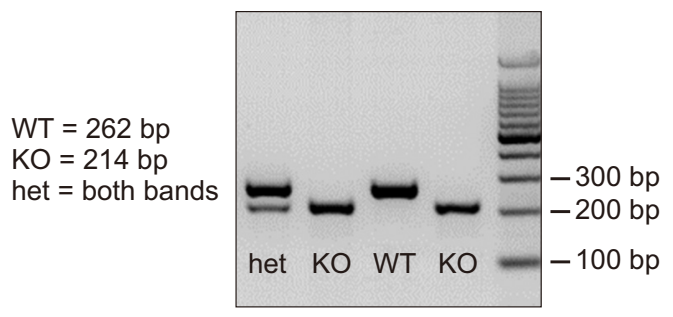

C

WT or Nrf2 KO

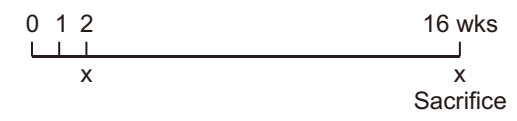

Group 1 (WT) or group 3 (KO) (control)

Group 2 (WT) or group 4 (KO) (AOM/DSS)

$\uparrow 2.5 \%$ DSS AOM

^: AOM injection (10 mg/kg) $\square: 2.5 \%$ DSS in drinking water $x$ : Sacrifice

Figure 1. Selection of nuclear factor erythroid 2-related factor 2 (Nrf2) knockout (KO) female mice using genotyping and a schematic illustration of the experiment. (A) Heterogeneous Nrf2 $\mathrm{KO}\left(\mathrm{Nrf}^{+-}\right)$male and female mice were mated and then homogeneous Nrf2 KO (Nrf2 ${ }^{-1}$ ) female mice were selected. (B) Genotyping of wildtype (WT) and Nrf2 KO mice. Representative agarose gel showing the PCR products. Nrf2 WT allele produces a 262-bp band, whereas the targeted allele produces a 214-bp band. Heterogeneous Nrf2 KO allele produces both, 214-bp and 262-bp bands. (C) Experimental scheme. WT and Nrf2 KO female mice were used in the azoxymethane (AOM)/ dextran sulfate sodium (DSS)-induced colitis-associated colorectal cancer (CRC) protocol. AOM $(10 \mathrm{mg} / \mathrm{kg})$ was injected to the mice on day 0 . One week later, DSS $(2.5 \%)$ was provided in the drinking water for one week. The mice were sacrificed at week 2 and 16 after AOM injection. $\widehat{\delta}$, male; o , female; +/+ and WT, wild-type; +/- and het, heterogeneous knockout; -/- and $\mathrm{KO}$, homogeneous knockout. 
were housed in cages at $23^{\circ} \mathrm{C}$ with a $12 / 12$-hour light/dark cycle under specific pathogen-free conditions. Genomic DNA (gDNA) obtained using DNeasy ${ }^{\circledR}$ Blood \& Tissue Kit (Qiagen Gmbh, Hilden, Germany) was used as the template DNA (listed in Table 1) and the PCR products were visualized under a ChemiDoc ${ }^{\mathrm{TM}}$ Imaging System (Bio-Rad Laboratories, Inc., Munich, Germany) (Fig. 1B). All animal procedures were approved by the Institutional Animal Care and Use Committee (IACUC) of the Seoul National University Bundang Hospital (BA1705-223/043-01) and performed in accordance with the ARRIVE (Animals Research: Reporting In Vivo Experiments) protocol.

\section{Induction of colitis-associated CRC in a mouse model}

For the induction of colitis-associated CRC, 2.5\% (w/v) DSS (Cat no 160110; MP Biomedicals, Solon, OH, USA) was supplied in the drinking water for 7 days, one week following the injection of AOM (10 mg/kg) (Cat no A5486; Sigma-Aldrich, St. Louis, MO, USA) counted as day $0[24,26]$. WT and Nrf2 $\mathrm{KO}$ female mice were randomized into the following groups. Group 1: WT control mice ( $n=11)$, Group 2: AOM/DSS-treated WT mice $(n=9-12)$, Group 3: Nrf2 KO control mice $(n=$ 10), Group 4: AOM/DSS-treated Nrf2 KO mice ( $n=13-16)$. The animals were euthanized by $\mathrm{CO}_{2}$ asphyxiation at week 2 (10 weeks of age) and 16 (24 weeks of age) after AOM injection (Fig. 1C).

\section{Evaluation of clinical symptoms}

Clinical symptoms were evaluated using the disease activity index (DAI), which includes loss of body weight, stool characterization, and hematochezia $[27,28]$. The DAI was scored by two researchers in a blinded manner as described elsewhere [24].

\section{Enumeration of lesions}

The colons were opened longitudinally, and stool was washed out with PBS. The colon length was measured from the cecum to the rectum using a ruler. Polypoid lesions with a diameter $\leq 2 \mathrm{~mm}$ or $>2 \mathrm{~mm}$ were independently counted by two researchers in a blinded manner $[27,28]$ as described previously [24]. The tumor incidence was determined as the percentage of mice bearing more than one tumor.

\section{Tissue processing and histopathology}

The extracted colon was divided into proximal and distal portions. The proximal colon up to $1.5 \mathrm{~cm}$ from the ileocecal valve, the rectum up to $1.5 \mathrm{~cm}$ from the anal verge, and colonic segments containing any gross polyps were fixed with phosphate-buffered formalin and embedded in paraffin. The sections $(5 \mathrm{~mm})$ were stained with hematoxylin and eosin (H\&E). The classification of adenoma and adenocarcino$\mathrm{ma}$, and the specification of the depth of adenocarcinoma invasion into the colonic tissues as mucosal or submucosal invasion were performed in a blinded manner [29]. The histological severity was assessed using a microscopic damage score reflecting colonic epithelial damage and depth of inflammatory cell infiltration [30].

Table 1. List of oligonucleotide sequence and their characteristics

\begin{tabular}{|c|c|c|}
\hline Gene & Sequence $\left(5^{\prime} \rightarrow 3^{\prime}\right)$ & Purpose \\
\hline Nrf2 WT & $\begin{array}{l}\text { F: GGA ATG GAAAAT AGC TCC TGC C } \\
\text { R: GCC TGA GAG CTG TAG GCC }\end{array}$ & Genotyping \\
\hline $\mathrm{Nrf2} \mathrm{KO}$ & R: GGG TTT TCC CAG TCA CGA & Genotyping \\
\hline Inos & $\begin{array}{l}\text { F: TGG TGG TGA CAA GCA CAT TT } \\
\text { R: AAG GCC AAA CAC AGC ATA CC }\end{array}$ & qRT-PCR \\
\hline Cox-2 & $\begin{array}{l}\text { F: TGA GTA CCG CAA ACG CTT CTC } \\
\text { R: TGG ACG AGG TTT TTC CAC CAG }\end{array}$ & qRT-PCR \\
\hline Tnf- $\alpha$ & $\begin{array}{l}\text { F: ACG GCA TGG ATC TCAAAG AC } \\
\text { R: GTG GGT GAG GAG CAC GTA GT }\end{array}$ & qRT-PCR \\
\hline II-6 & $\begin{array}{l}\text { F: CTG CAA GAG ACT TCC ATC CAG TT } \\
\text { R: GAA GTA GGG AAG GCC GTG G }\end{array}$ & qRT-PCR \\
\hline Ho-1 & $\begin{array}{l}\text { F: CCT CAC TGG CAG GAA ATC ATC } \\
\text { R: CCT CGT GGA GAC GCT TTA CAT A }\end{array}$ & qRT-PCR \\
\hline Gclc & $\begin{array}{l}\text { F: ACA TCT ACC ACG CAG TCA AGG ACC } \\
\text { R: CTC AAG AAC ATC GCC TCC ATT CAG }\end{array}$ & qRT-PCR \\
\hline Gclm & $\begin{array}{l}\text { F: GCC ACC AGA TTT GAC TGC CTT TG } \\
\text { R: TGC TCT TCA CGA TGA CCG AGT ACC }\end{array}$ & qRT-PCR \\
\hline Gapdh & $\begin{array}{l}\text { F: TTC ACC ACC ATG GAG AAG GC } \\
\text { R: GGC ATG GAC TGT GGT CAT GA }\end{array}$ & qRT-PCR \\
\hline
\end{tabular}

Nrf2, nuclear factor erythroid-derived 2-related factor 2; WT, wild type; KO, knockout; F, forward; R, reverse; Inos, inducible nitric oxide synthase; Cox-2, cyclooxygenase-2; Tnf- $\alpha$, tumor necrosis factor-alpha; II-6, interleukin 6; Ho-1, heme oxygenase 1; Gclc, glutamatecysteine ligase catalytic subunit; Gclm, glutamate-cysteine ligase modifier subunit; Gapdh, glyceraldehyde-3-phosphate dehydrogenase. 


\section{Measurement of inflammatory cytokines}

The levels of myeloperoxidase (MPO), IL-6, and IL-1 $\beta$ in the colonic tissues were measured using a mouse MPO ELISA kit (Cat no HK210; Hycult Biotechnology, Uden, The Netherlands), a mouse IL-6 Quantikine ELISA kit (Cat no M6000B; R\&D Systems Inc., Minneapolis, MN, USA), and a mouse IL-1 $\beta / I L-1 F 2$ Quantikine ELISA kit (Cat no MLB00C; R\&D Systems Inc.), respectively, according to the manufacturer's instructions. All assays were performed in triplicate.

\section{Quantitative real-time polymerase chain reaction (qRT-PCR)}

Total RNA was extracted from the colon tissues using TRIzol ${ }^{\circledR}$ reagent (Invitrogen, Carlsbad, CA, USA). For qRT-PCR, 2 $\mu \mathrm{g}$ of total RNA was reverse transcribed using High Capacity cDNA Reverse Transcription kit according to the manufacturer's instructions (Applied Biosystems, Foster City, CA, USA). The cDNA was used to perform qRT-PCR using specific primers (listed in Table 1) in a ViiA7 instrument (Applied Biosystems). The expression levels were normalized to that of Gapdh.

\section{Western blot analysis}

Colon tissue was lysed with RIPA buffer (Cell Signaling Technology, Beverly, MA, USA) containing protease and phosphatase inhibitors. Total protein was separated by SDSPAGE and transferred to a polyvinylidene difluoride (PVDF) membrane. Western blot analysis was performed with specific primary antibodies (listed in Table 2). The signals were then detected with an enhanced chemiluminescence (ECL) kit (GE Healthcare Biosciences, Buckinghamshire, UK). The band intensity was quantified by densitometric analysis using the ImageJ software (National Institutes of Health, Bethesda, MD, USA).

\section{Statistical analysis}

All statistical analyses were conducted using GraphPad Prism, version 5.01 (GraphPad Software, Inc., San Diego, CA, USA) and PASW Statistics for Windows, version 18.0 (IBM Corp., Armonk, NY, USA). Data are expressed as the

Table 2. List of antibodies and their characteristics

\begin{tabular}{lll}
\hline \multicolumn{1}{c}{ Antigen } & \multicolumn{1}{c}{ Antibody (Cat no.) } & \multicolumn{1}{c}{ Dilution } \\
\hline iNOS & BD Biosciences (\#610328) & WB $(1: 500)$ \\
NQO1 & Abcam (ab34173) & WB $(1: 1,000)$ \\
GCLC & Abcam (ab41463) & WB $(1: 1,000)$ \\
ER $\beta$ & Abcam (ab3576) & WB $(1: 500)$ \\
15-PGDH & Cayman Chemical (\#160615) & WB $(1: 1,000)$ \\
$\beta-A c t i n$ & Santa Cruz Biotechnology & WB $(1: 2,000)$ \\
& $($ sc47778) &
\end{tabular}

WB, Western blot; iNOS, inducible nitric oxide synthase; NQO1, $\mathrm{NAD}(\mathrm{P}) \mathrm{H}$ : quinone dehydrogenase 1; GCLC, glutamate-cysteine ligase catalytic subunit; ER $\beta$, estrogen receptor beta; $15-\mathrm{PGDH}$, 15 -hydroxyprostaglandin dehydrogenase; $\beta$-Actin, beta-actin. mean \pm SEM. Statistical significance was examined with the Mann-Whitney test or Fisher's exact test. $P<0.05$ was considered statistically significance.

\section{RESULTS}

Increased colonic epithelial damage and shortening of colon length by Nrf2 KO in female mice at the colitis stage

To investigate the potential role of Nrf2 in AOM/DSS-induced inflammation and tumorigenesis in female mice, a total of four experimental groups were prepared as presented in Figure 1C. Two WT groups comprised female control mice (Group 1) and AOM/DSS-treated female mice (Group 2). In Nrf2 KO groups, two groups also comprised female control mice (Group 3) and AOM/DSS-treated female mice (Group 4).

First, we measured the colitis-associated symptoms including DAI, colonic epithelial damage, and colon length shortening. The DAI was calculated by summing the body weight change, stool consistency, and rectal bleeding, and by dividing the result by three. In the AOM/DSS-treated animal model, body weight loss is a sensitive indicator of colitis severity [31], which is linked to colon tumorigenesis. The body weight loss caused by AOM/DSS treatment was observed in both WT and Nrf2 KO groups without the effect of Nrf2 $\mathrm{KO}$ at week 2 (Fig. 2A and 2B). However, at week 16, significant body weight loss was observed in both Nrf2 KO control and AOM/ DSS groups (Fig. 2A and 2C, $P=0.040$ for WT control vs KO control and $P=0.005$ for WT AOM/DSS vs. KO AOM/DSS). After careful analysis of the body weight changes, the DAI score increased by AOM/DSS treatment was found not to be affected by Nrf2 KO at week 2 and 16 (Fig. 2D-2F). Representative histopathological images (Fig. 2G) and microscopic damage scores (Fig. $2 \mathrm{H}$ ) indicated that colonic epithelial damage induced by AOM/DSS treatment was more evident in the $\mathrm{Nrf} 2 \mathrm{KO}$ group as evidenced by higher inflammatory cell infiltration and strong cryptic damage than in WT mice (Fig. $2 \mathrm{G}$ and $2 \mathrm{H}, P<0.001$ for WT AOM/DSS vs. KO AOM/DSS). Interestingly, colon length was shortened by $\mathrm{Nrf} 2 \mathrm{KO}$ in both the control and AOM/DSS-treated groups compared to WT mice, but showed significant differences only in the control group (Fig. 2l, $P<0.001$ for WT control vs. Nrf2 KO control). As a result, colon length shortening induced by AOM/DSS treatment was not affected by Nrf2 KO (Fig. 2l). These results suggest that Nrf2 plays a protective role against inflammation in both the intact and colitis stages.

\section{Attenuation of colitis-associated tumorigenesis by Nrf2 KO in female mice at tumorigenesis stage}

To determine the effect of Nrf2 on colitis-associated tumorigenesis in females, we counted the tumor number by macroscopic assessment, assessing both the size and the location, at week 16. Tumors were well developed at week 16 in both 


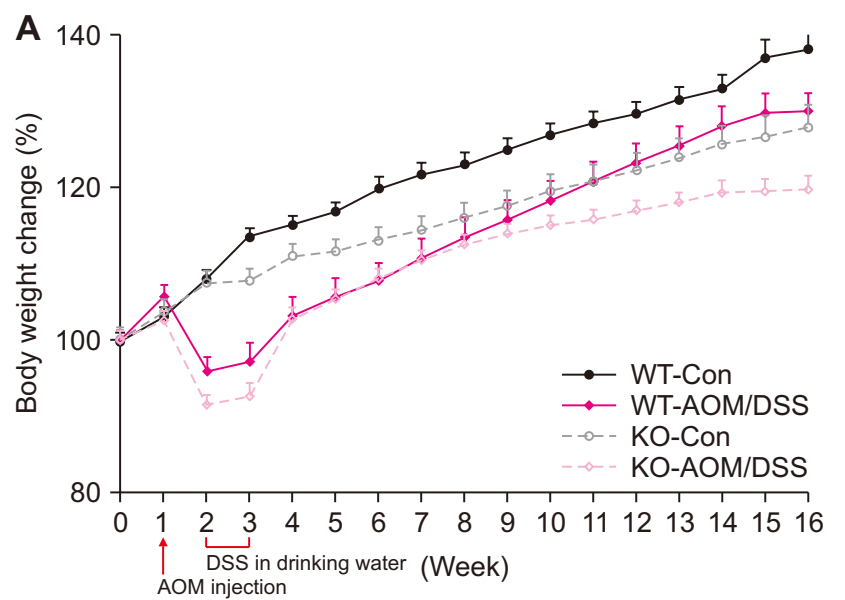

D

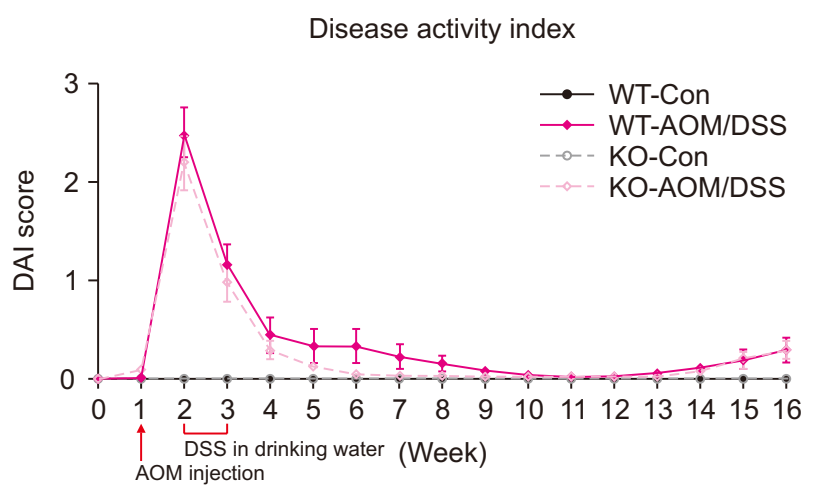

G

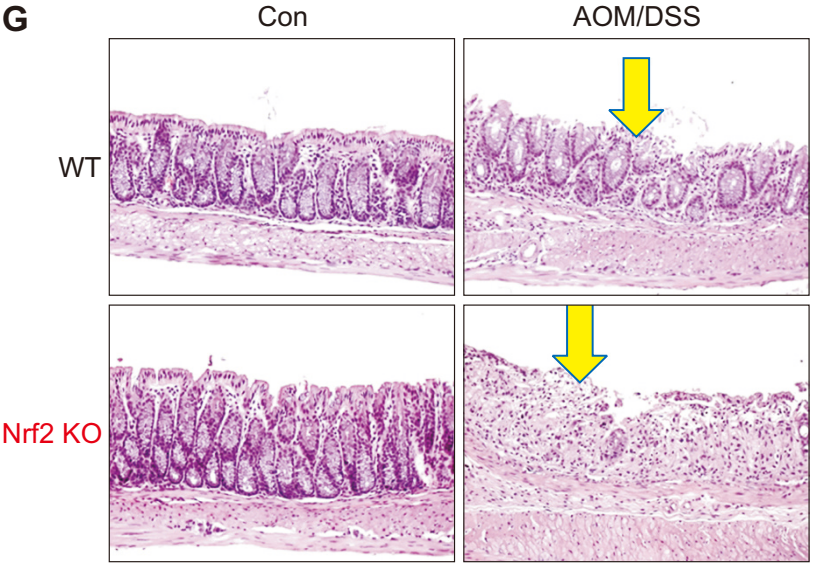

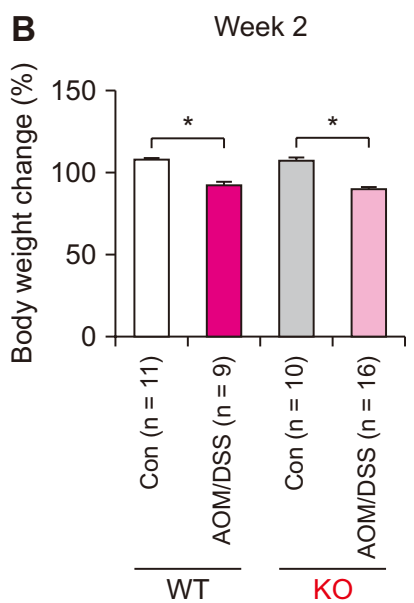
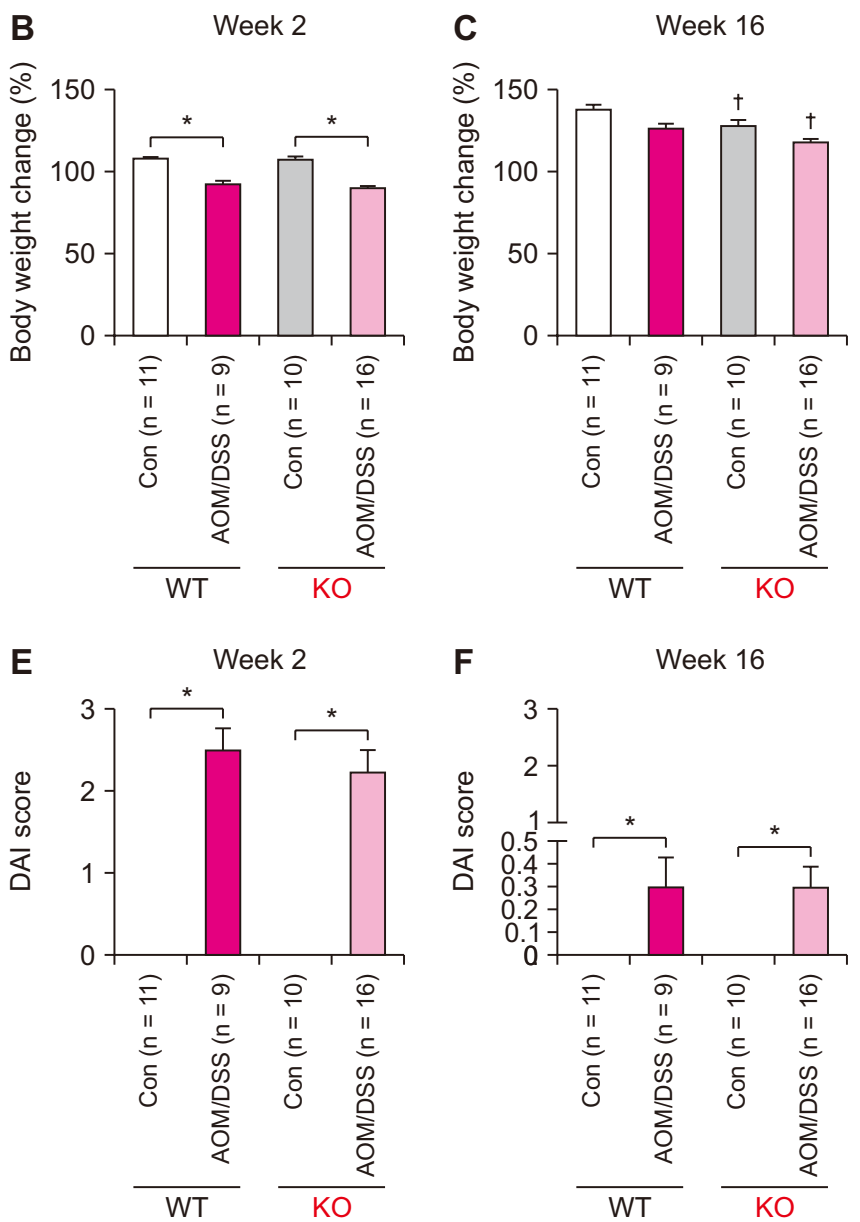

$\mathbf{F}$

Week 16
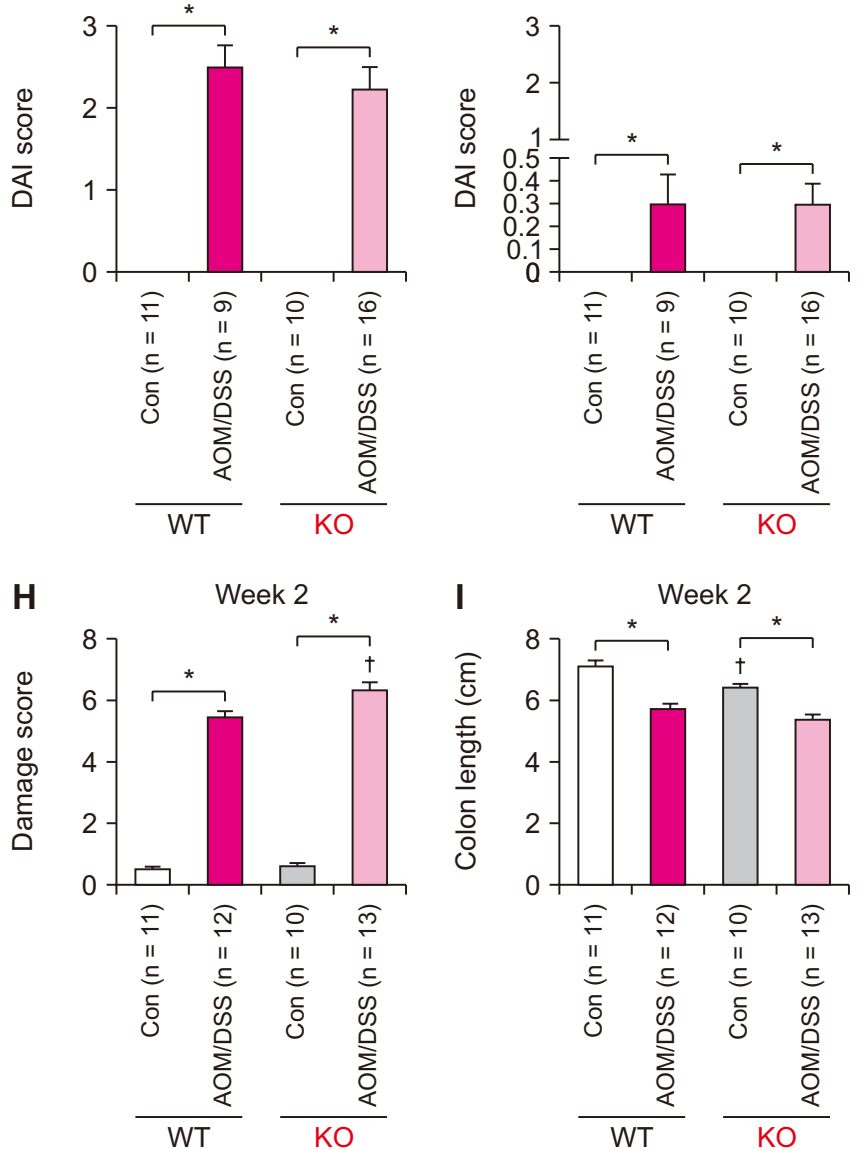

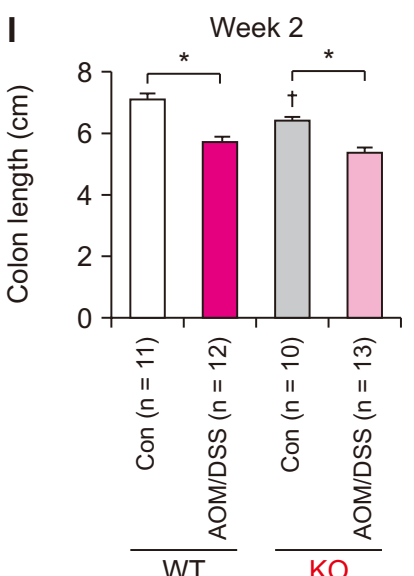

Figure 2. Effect of nuclear factor erythroid 2-related factor 2 (Nrf2) KO on phenotypic changes and inflammatory index in AOM/DSSinduced colitis. (A-C) Body weight chages during the experimental period (A), at week 2 (B), and at week 16 (C). (D-F) DAl score during experimental period (D), at week $2(\mathrm{E})$, and at week $16(\mathrm{~F})$. (G) Increase of AOM/DSS-induced inflammatory cell infiltration by Nrf2 KO in female mice. Representative histological images of colonic mucosa following H\&E staining at week 2. Magnification, $\times 100$. The mucosa was normal in WT and Nrf2 KO control mice. However, near-total loss of crypt and severe inflammatory cell infiltration of the colonic mucosa (arrows) were observed in both AOM/DSS-treated WT and Nrf2 KO male mice. $(\mathrm{H})$ The microscopic damage score induced by AOM/DSS treatment was weakened in AOM/ DSS-induced Nrf2 KO male mice. (I) Colon length at week 2. WT, wild-type; KO, knockout; AOM, azoxymethane; DSS, dextran sodium sulfate; DAI, disease activity index. ${ }^{*} P<0.05$ between two groups; ${ }^{\dagger} P<0.05$ for WT vs. Nrf2 KO. 


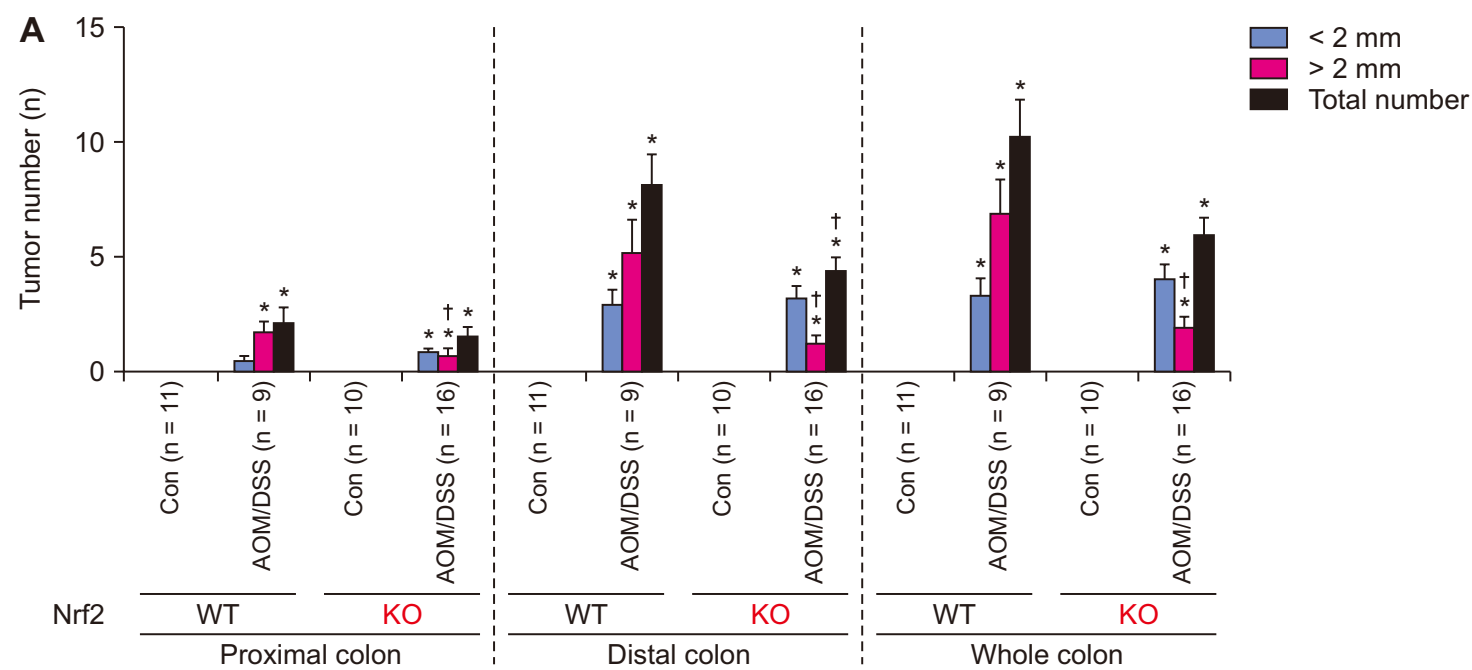

B Con AOM/DSS
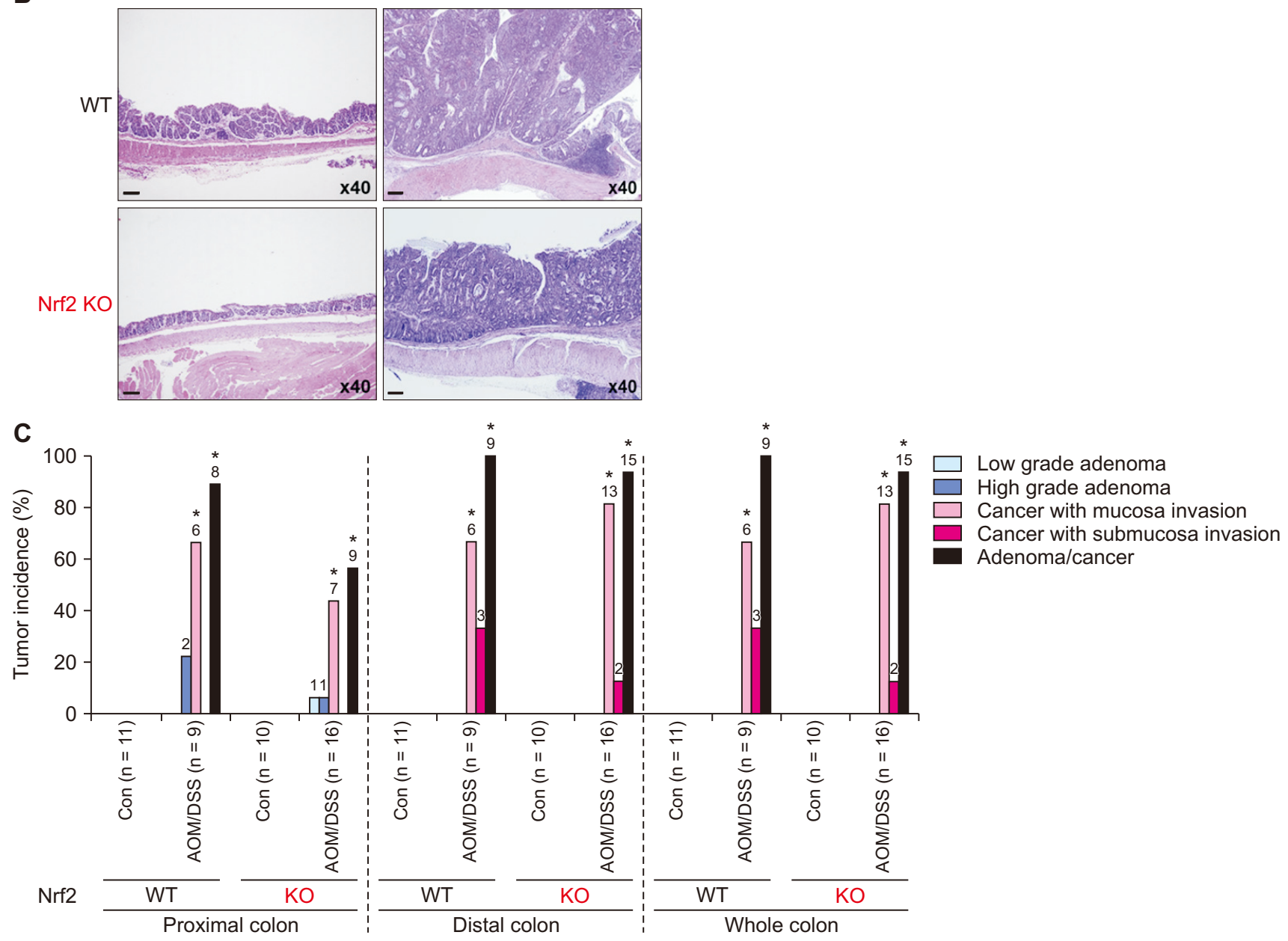

Figure 3. Effect of nuclear factor erythroid 2-related factor 2 (Nrf2) KO on the multiplicity of colorectal cancer (CRC) at weeks 16. (A) Average number of tumors and size distribution in the proximal, distal, and whole colon in each group sacrificed at week 16 following AOM injection. (B) Representative histological images following H\&E staining at week 16. Magnification, $\times 40$. Scale bar, $200 \mu \mathrm{m}$ (C) Quantification of adenoma/ adenocarcinoma incidence and invasion in each group by microscopic evaluation of the colonic tissues at the tumorigenesis stage (at week 16). The case number is marked on the top of the bar. WT, wild-type; KO, knockout; AOM, azoxymethane; DSS, dextran sodium sulfate; Con., control. ${ }^{*} P<0.05$ for Con. vs. AOM/DSS; ${ }^{\dagger} P<0.05$ for WT vs. Nrf2 KO. 


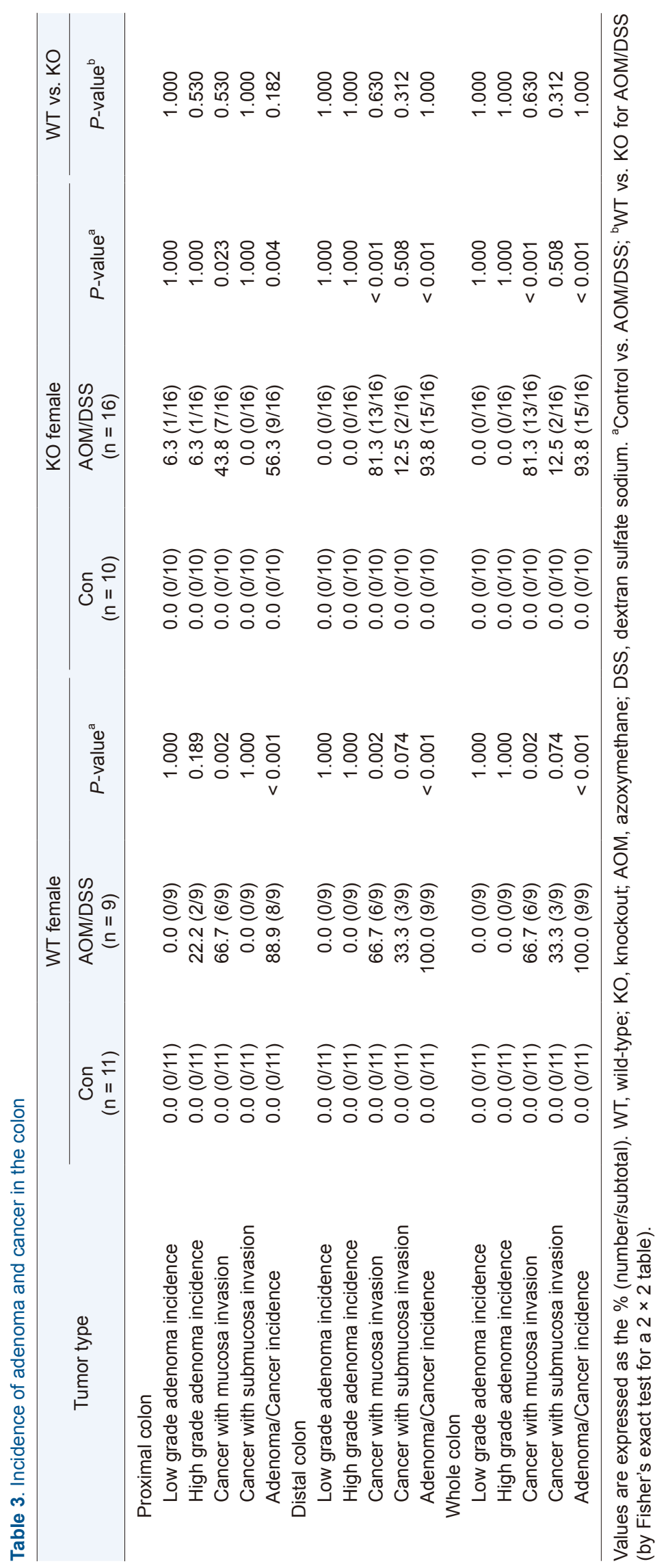



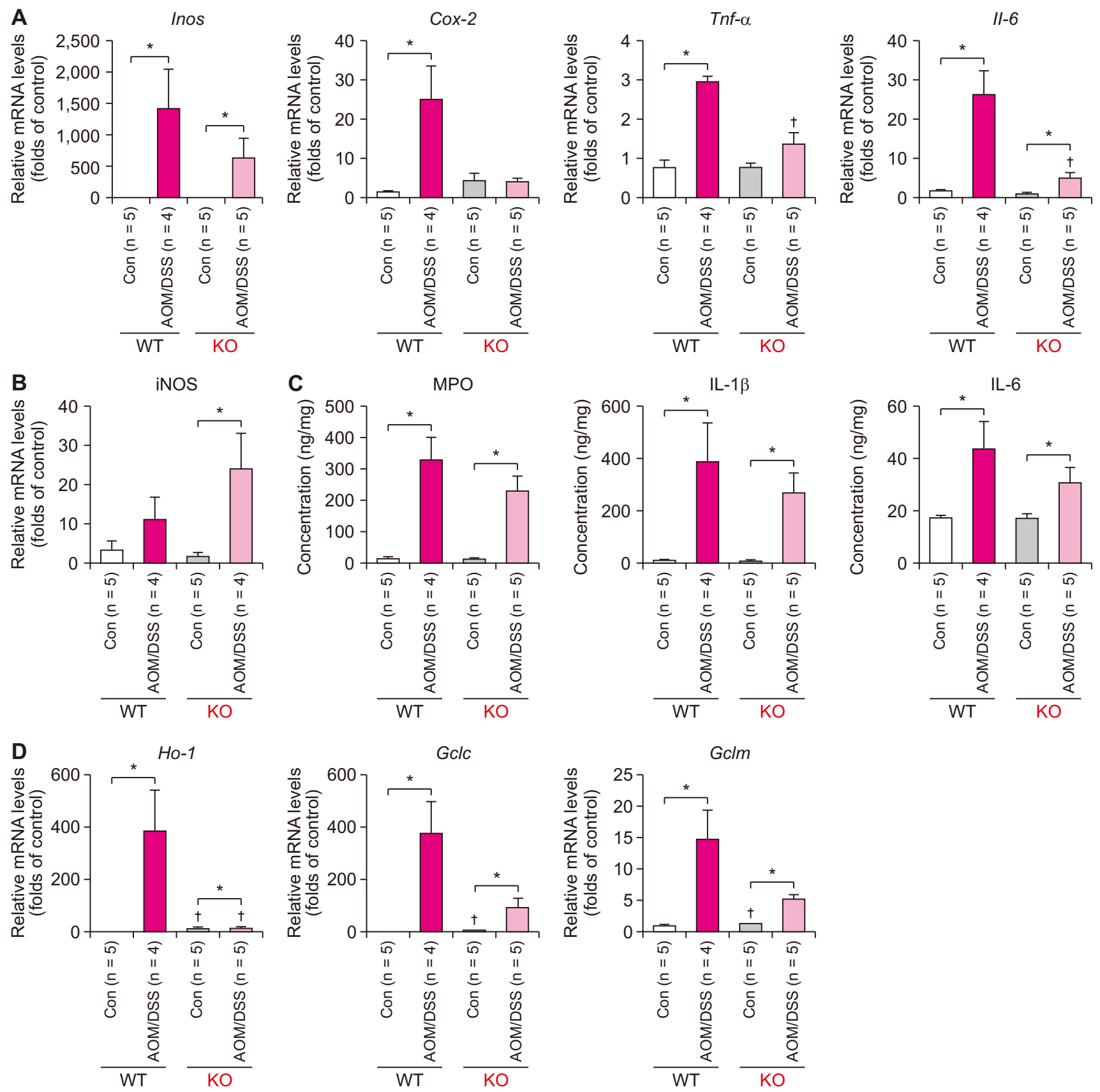

Figure 4. Effect of nuclear factor erythroid 2-related factor 2 ( $\mathrm{Nrf2}$ ) KO on expression/production of pro-inflammatory enzymes and cytokines and anti-oxidant enzymes in colonic tissues at week 2. (A) The mRNA expression of pro-inflammatory genes such as Inos, Cox2, Tnf- $\alpha$, and II-6 determined qRT-PCR analysis. (B) iNOS protein expression measured Western blot analysis (60 $\mu \mathrm{g}$ of protein). (C) MPO, IL-1 $\beta$, and IL-6 production in colon tissue measured by ELISA. (D) The mRNA expression of anti-oxidant enzyme genes such as Ho-1, Gclc, and Gclm as assessed qRT-PCR analysis. WT, wild-type; KO, knockout; AOM, azoxymethane; DSS, dextran sodium sulfate; iNOS, inducible nitric oxide synthase; COX-2, cyclooxygenase-2; IL-6, interleukin 6; MPO, myeloperoxidase; HO-1, heme oxygenase 1; GCLC, glutamate-cysteine ligase catalytic subunit; GCLM, glutamate-cysteine ligase modifier subunit; Con., control. ${ }^{*} P<0.05$ for Con. vs. AOM/DSS; ${ }^{\dagger} P<0.05$ for WT vs. Nrf2 KO.

the proximal and distal regions of colon in AOM/DSS-treated WT and Nrf2 KO mice (Fig. 3A). Interestingly, tumor numbers, especially those $>2 \mathrm{~mm}$ in size, were significantly lower in Nrf2 $\mathrm{KO}$ mice than in WT mice both in proximal and distal regions of colon (Fig. 3A, $P=0.023$ for proximal colon and $P$
$=0.002$ for distal colon). Representative histopathological images are shown in Figure 3B. The incidence of microscopic colonic neoplasms such as adenoma and cancer is summarized in Table 3. In the proximal colon, the total incidence of adenoma/cancer formed by AOM/DSS treatment was de- 
creased in Nrf2 KO (56.3\%) mice than in WT (88.9\%) mice, and in particular, the incidence of mucosal invasive cancer was reduced by approximately $23 \%(66.7 \%$ in WT and $43.8 \%$ in $\mathrm{KO}$ ) (Fig. $3 \mathrm{C}$ ). The total incidence of adenoma/cancer in the distal colon was not affected by Nrf2 KO (93.8\%) compared with WT (100\%) mice, whereas submucosal invasive cancer decreased approximately 2.7-fold in Nrf2 KO (12.5\%) mice compared with WT (33.3\%) animals (Fig. 3C). These findings provide evidence that Nrf2 is involved in tumor promotion/progression in colitis-associated tumorigenesis.

\section{Effect of Nrf2 KO on the expression of the pro-inflammatory mediators and anti-oxidant genes at colitis stage}

To further assess the effect of Nrf2 $\mathrm{KO}$ on inflammatory mediators at the molecular level, NF-кB-mediated expression of pro-inflammatory enzymes and cytokines, and their gene levels were measured in colon tissue at week 2 . The mRNA expression levels of inducible nitric oxide synthase (Inos), cyclooxygenase-2 (Cox-2), tumor necrosis factor-alpha (Tnf- $\alpha$ ), and interleukin 6 (II-6) were strongly elevated by AOM/DSS treatment in WT mice (Fig. 4A). However, in the Nrf2 KO group, the AOM/DSS-induced mRNA expression of pro-inflammatory genes tended to decrease compared to the WT group, showing a significant difference only in Tnf- $\alpha$ and II-6 (Fig. 4A, $P=0.002$ for Tnf- $\alpha$ and $P<0.001$ for II-6). The protein expression of iNOS induced by AOM/DSS treatment was significantly increased in the Nrf2 KO group, but not in the WT mice (Fig. 4B). Next, we performed ELISA to measure the concentration of the pro-inflammatory mediators such as MPO, IL-1 $\beta$, and IL-6. After AOM/DSS treatment, colonic MPO, IL-1 $\beta$, and IL- 6 levels were elevated to a similar extent in both the WT and Nrf2 KO groups (Fig. 4C).

We further measured mRNA expression of the Nrf2-mediated anti-oxidant enzymes at week 2 . The mRNA expression of $\mathrm{Ho}-1, \mathrm{Gclc}$, and $\mathrm{Gclm}$ increased by AOM/DSS treatment in WT mice was strongly attenuated in the Nrf2 KO group, with a significant difference observed only in $\mathrm{Ho}-1$ (Fig. 4D, $P<$ 0.001 for $\mathrm{Ho}-1$ ).

\section{Effect of Nrf2 KO on the expression of the pro-inflammatory mediators and anti-oxidant genes at the tumorigenesis stage}

At week 16, the mRNA expression levels of Inos, Cox-2, Tnf- $\alpha$, and II- 6 genes were strongly elevated by AOM/DSS treatment in both the WT and Nrf2 KO groups (Fig. 5A). The AOM/DSS-induced Inos and Cox-2 mRNA expression showed a significant decrease in the Nrf2 KO group compared to WT mice (Fig. 5A, $P=0.008$ for Inos and $P=0.005$ for Cox-2). AOM/DSS-induced iNOS protein expression was significantly increased in the Nrf2 KO group, but not in the WT group (Fig. 5B). The concentrations of colonic MPO, IL$1 \beta$, and IL- 6 induced by AOM/DSS treatment were elevated in both the WT and Nrf2 KO groups, but their levels were low- er more than 2-folds in the Nrf2 $\mathrm{KO}$ group compared to the WT mice (Fig. 5C).

We further measured mRNA and protein expression of the Nrf2 target genes encoding anti-oxidant enzymes at week 16. The mRNA expression of $\mathrm{Ho}-1, \mathrm{Gclc}$, and $\mathrm{Gclm}$ increased by AOM/DSS treatment in WT mice was strongly attenuated in the Nrf2 KO group, showing a significant difference only in Ho-1 (Fig. 5D, $P<0.001$ for Ho-1). The protein expression of NQO1 and GCLC induced by AOM/DSS treatment also showed lower levels in the Nrf2 KO group compared to WT mice (Fig. 5E).

To figure out the molecular signatures on Nrf2-associated tumorigenesis in female mice, we analyzed the colonic expression of ER $\beta$ and 15-hydroxy prostaglandin dehydrogenase (15-PGDH). There was no change in the protein expression of ER $\beta$ by AOM/DSS treatment in WT mice, but the expression of ER $\beta$ was significantly decreased by AOM/DSS treatment in the Nrf2 KO group (Fig. 5F, $P=0.006$ for control vs. AOM/DSS in Nrf2 KO). In particular, the protein expression of ER $\beta$ was significantly higher in the Nrf2 KO control group compared to WT mice (Fig. 5F, $P=0.016$ for WT control vs. Nrf2 KO control). Interestingly, the protein expression of 15-PGDH in the Nrf2 KO group was regulated in a manner opposite to that of ER $\beta$ (Fig. 5G, $P=0.045$ for control vs. AOM/DSS in Nrf2 KO and $P=0.009$ for WT control vs. Nrf2 $\mathrm{KO}$ control).

\section{DISCUSSION}

Our results showed that AOM/DSS-mediated colonic epithelial damages were significantly worse in Nrf2 KO female mice than in WT at the colitis stage (week 2). However, at the tumorigenesis stage (week 16), the number of tumors exceeding $2 \mathrm{~mm}$ in size was significantly lower in both the proximal and distal colons in Nrf2 KO compared to WT female mice. Furthermore, the overall adenoma/cancer incidence of the proximal colon and submucosal invasive cancer of the distal colon was decreased in Nrf2 KO mice than in WT animals. Interestingly, the mRNA or protein expression of $\mathrm{NF}-\kappa \mathrm{B}$-related mediators and Nrf2-related antioxidant enzymes was significantly lower in Nrf2 KO mice, while the protein expression level of 15-PGDH was higher in Nrf2 KO than in WT mice. These results suggest that Nrf2 acts differentially, in a way that it exerts an anti-inflammatory effect in the colitis stage and a carcinogenic effect in the tumorigenesis stage.

Nrf2 is a key modulator of the adaptive response to a variety of environmental and endogenous stresses [32-36]. Recently, there are several reports that Nrf2 contributes to weight gain. The body weight of adult Nrf2 KO mice was lower compared to WT mice fed a normal diet [37]. Nrf2 was involved in weight gain in male mice during space travel by maintaining homeostasis of white adipose tissue [38]. Decreased expression of peroxisome proliferator-activated receptor $\gamma$ due to Nrf2 deficiency prevented weight gain and 
obesity from a high fat diet and environmental stress caused by space travel $[37,38]$. In our previous study, the body weight of male control mice was not affected by Nrf2 KO at week 16 [24]. In the present study, however, the body weight of the Nrf2 KO female control or AOM/DSS-treated group was significantly lower compared to that of the WT group at
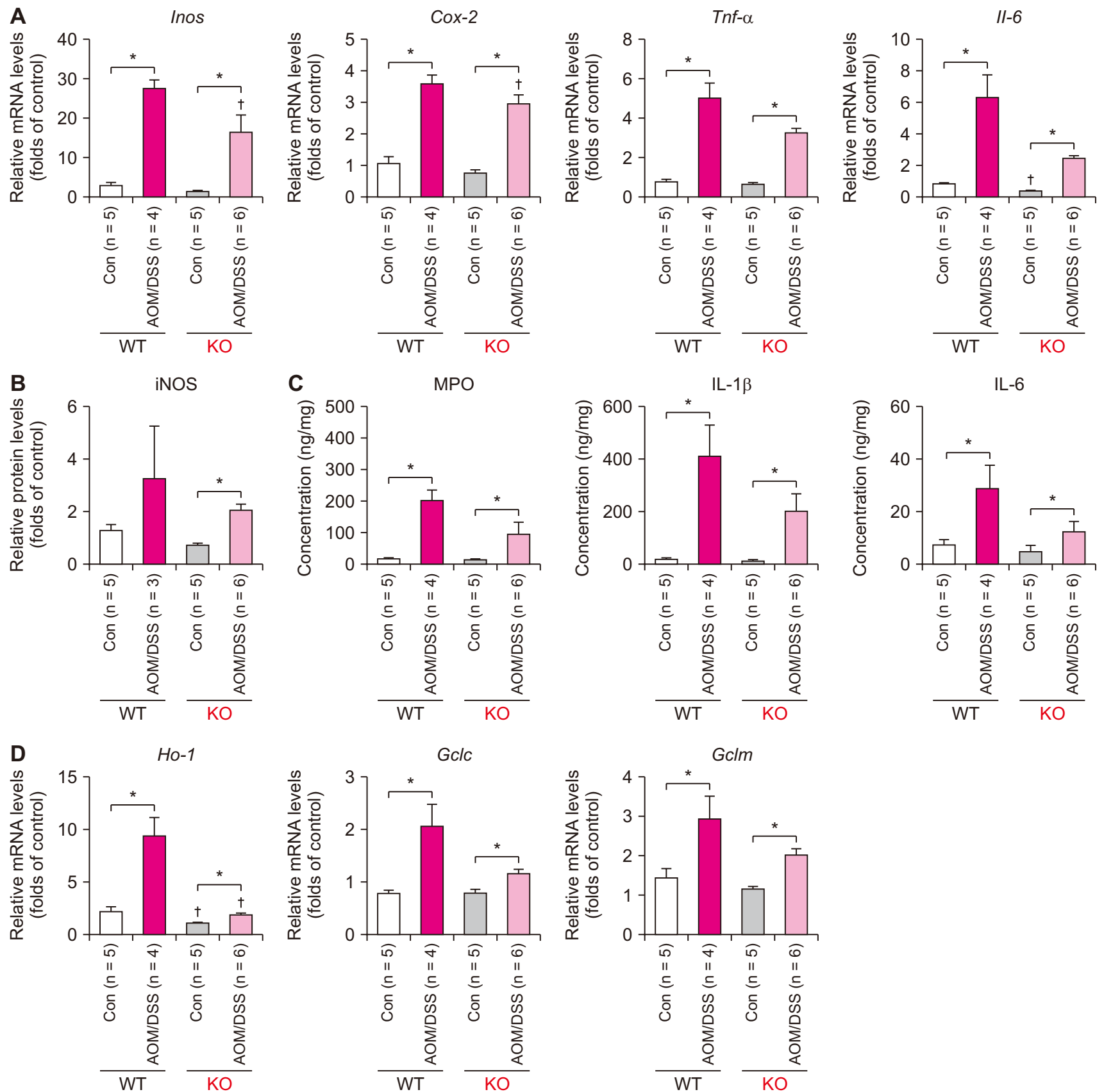

Figure 5. Effect of nuclear factor erythroid 2-related factor 2 (Nrf2) KO on the mRNA or protein expression levels of pro-inflammatory and anti-oxidant enzyme genes in colonic tissues at week 16. (A) The mRNA expression of pro-inflammatory genes such as Inos, Cox-2, Tnf- $\alpha$, and II- 6 by qRT-PCR analysis. (B) iNOS protein expression by Western blot analysis (60 $\mu \mathrm{g}$ of protein). (C) MPO, IL-1 $\beta$, and IL- 6 concentration in colon tissue by ELISA. (D) The mRNA expression of anti-oxidant enzyme genes such as Ho-1, Gclc, and Gclm by qRT-PCR analysis. (E) The protein expression of anti-oxidant enzymes such as NQO1 and GCLC by Western blot analysis (30 $\mu \mathrm{g}$ of protein). (F, G) The protein expression of ER $\beta(F)$ and 15-PGDH (G) by Western blot analysis (60 $\mu$ g of protein). WT, wild-type; KO, knockout; AOM, azoxymethane; DSS, dextran sodium sulfate; iNOS, inducible nitric oxide synthase; COX-2, cyclooxygenase-2; IL-6, interleukin 6; MPO, myeloperoxidase; HO-1, heme oxygenase 1; GCLC, glutamate-cysteine ligase catalytic subunit; GCLM, glutamate-cysteine ligase modifier subunit; NQO1, NAD(P)H: quinone dehydrogenase 1; ER $\beta$, estrogen receptor beta; 15-PGDH, 15-hydroxyprostaglandin dehydrogenase; Con., control. ${ }^{*} P<0.05$ for Con. vs. AOM/DSS; ${ }^{\dagger} P<0.05$ for WT vs. $\mathrm{Nrf} 2 \mathrm{KO}$ 

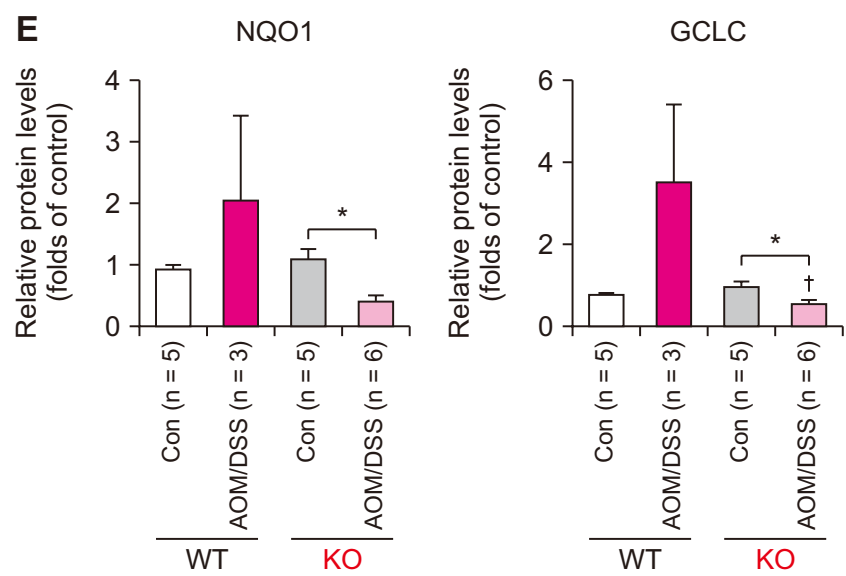

Figure 5. Continued.

week 16. Several studies have shown that Nrf2 KO mice are more susceptible to inflammatory diseases $[39,40]$. Khor et al. reported that mRNA and protein expression of COX-2 and iNOS induced by AOM/DSS treatment increased in colon tissue of Nrf2 KO male mice compared to WT animals [41]. A study using peritoneal macrophages prepared from WT male and Nrf2 KO male mice showed that iNOS protein expression was more strongly inhibited by phenethyl isothiocyanate and curcumin treatment in WT than in Nrf2 KO mice [32]. At week 2 , colon length shortening and colonic epithelial damages were also affected similarly to that of Nrf2 KO-mediated weight loss. In males, the colon length of the control group and colonic epithelial damages induced by AOM/DSS were not affected by Nrf2 KO [24]. However, females were easily affected by $\mathrm{Nrf2} \mathrm{KO}$, as revealed by shortening of the colon length and increased colon epithelial damage. At the molecular level, the protein expression of the proinflammatory mediator iNOS induced by AOM/DSS treatment was lower in WT females than in WT males [24]; however, sex differences in iNOS expression disappeared by Nrf2 KO. These results indicate that the contribution of Nrf2 to homeostasis maintenance and inflammatory defense mechanisms is greater in female mice than in males. It is speculated that female mice are more susceptible to Nrf2 KO than males.

Recently, Nrf2 was known to be involved in maintaining cancer cell growth and invasion by metabolic reprogramming, inhibiting cancer cell apoptosis, and enhancing the ability of cancer stem cells to self-renew in the tumorigenesis stage. More importantly, aberrant activation of Nrf2 was associated with chemoresistance of cancer cells and poor prognosis [42]. In the previous study, we also observed that Nrf2 and Nrf2-related antioxidant genes were highly expressed in AOM/DSS-treated male and female mice, which accelerated cancer development [23]. Therefore, we expected that tumorigenicity would be weaker in the Nrf2 $\mathrm{KO}$ group than in WT mice. As expected, the incidence of tumors was lower in the Nrf2 KO female mice than in the WT females, especially
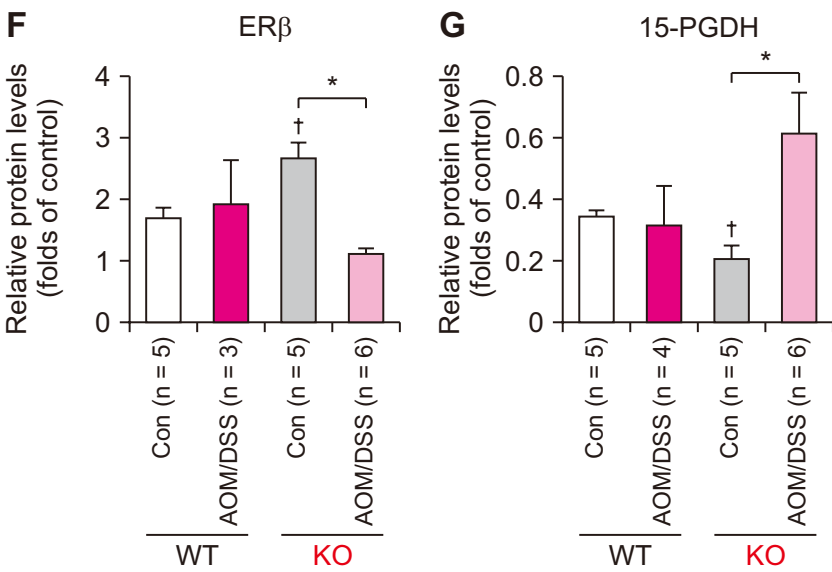

those with tumor sizes greater than $2 \mathrm{~mm}$. In particular, only the incidence of submucosal invasive cancer at the distal colon was reduced by Nrf2 $\mathrm{KO}$ in females. In the previous study, the tumor incidence in male mice was not affected by $\mathrm{Nrf2} \mathrm{KO}$, so there was no difference from the tumor incidence in WT mice [24]. Interestingly, the anti-tumorigenic effect of $17 \beta$-estradiol was stronger in the AOM/DSS-treated Nrf2 KO group than in WT male mice [24].

Several studies have demonstrated the tumor suppressor function of 15-PGDH, an enzyme that converts prostaglandin $E_{2}$ into an inactive metabolite [43-45]. The expression of 15$\mathrm{PGDH}$ was strongly reduced in various human malignancies such as colon cancer, lung cancer, and gastric cancer compared to normal tissues [46,47]. 15-PGDH is abundantly expressed in the normal colonic mucosa, and loss of 15$\mathrm{PGDH}$ is associated with CRC development $[46,48]$. In the present study, there was no change in the protein expression of 15-PGDH by AOM/DSS treatment compared to the female control in the 16-week WT group, but the expression of 15$\mathrm{PGDH}$ was strongly increased in the AOM/DSS-treated Nrf2 $\mathrm{KO}$ female group compared to its control. In the male group, the expression of 15-PGDH was strongly inhibited in both WT and Nrf2 KO mice by AOM/DSS treatment, and there was no change by $\mathrm{Nr} 2 \mathrm{KO}$ (data not shown). Interestingly, there was no change in the expression of 15-PGDH and ER $\beta$ by AOM/ DSS treatment in the WT female mice, but it was observed that protein expression of 15-PGDH and ER $\beta$ was oppositely regulated in the Nrf2 $\mathrm{KO}$ female group. Taken together our results regarding $\mathrm{ER} \beta$ and 15-PGDH in the Nrf2 KO mice suggest that 15-PGDH might contribute to the suppression of colitis-associated tumorigenesis in Nrf2 KO female mice. However, further experiments are needed to clarify this supposition.

In conclusion, our study shows the possibility that Nrf2 KO suppresses the colitis-associated tumorigenesis by upregulating tumor suppressor 15-PGDH in females. The elevated Nrf2 levels are associated with the development of aggressive CRC, and 15-PGDH may be a useful therapeutic target 
depending on sex in CRC patients.

\section{ACKNOWLEDGMENTS}

We are grateful to Prof. Young-Joon Surh (Seoul National University College of Pharmacy, Seoul, Korea) for providing heterozygous Nrf2 knockout $\left(\mathrm{Nrf}^{+/-}\right)$mice. This research was supported by a grant from the National Research Foundation of Korea (NRF) funded by the government of the Republic of Korea (2019R1A2C2085149).

\section{CONFLICTS OF INTEREST}

No potential conflicts of interest were disclosed.

\section{ORCID}

Chin-Hee Song, https://orcid.org/0000-0002-3489-5944

Nayoung Kim, https://orcid.org/0000-0002-9397-0406

Ryoung Hee Nam, https://orcid.org/0000-0002-6515-4540

Soo In Choi, https://orcid.org/0000-0001-6625-7608

Changhee Kang, https://orcid.org/0000-0002-3955-2764

Jae Young Jang, https://orcid.org/0000-0003-2463-7746

Heewon Nho, https://orcid.org/0000-0002-0324-0427

Eun Shin, https://orcid.org/0000-0002-9627-7914

Ha-Na Lee, https://orcid.org/0000-0001-5063-6689

\section{REFERENCES}

1. Siegel RL, Miller KD, Fuchs HE, Jemal A. Cancer statistics, 2021. CA Cancer J Clin 2021;71:7-33.

2. Hong S, Won YJ, Park YR, Jung KW, Kong HJ, Lee ES. Cancer statistics in Korea: incidence, mortality, survival, and prevalence in 2017. Cancer Res Treat 2020;52:335-50.

3. Kim SE, Paik HY, Yoon H, Lee JE, Kim N, Sung MK. Sex- and gender-specific disparities in colorectal cancer risk. World J Gastroenterol 2015;21:5167-75.

4. Tonelli C, Chio IIC, Tuveson DA. Transcriptional regulation by Nrf2. Antioxid Redox Signal 2018;29:1727-45.

5. Rojo de la Vega M, Dodson M, Gross C, Mansour HM, Lantz RC, Chapman E, et al. Role of Nrf2 and autophagy in acute lung injury. Curr Pharmacol Rep 2016;2:91-101.

6. McMahon M, Itoh K, Yamamoto M, Hayes JD. Keap1-dependent proteasomal degradation of transcription factor Nrf2 contributes to the negative regulation of antioxidant response element-driven gene expression. J Biol Chem 2003;278:21592-600.

7. Thimmulappa RK, Mai KH, Srisuma S, Kensler TW, Yamamoto M, Biswal S. Identification of Nrf2-regulated genes induced by the chemopreventive agent sulforaphane by oligonucleotide microarray. Cancer Res 2002;62:5196-203.

8. Kundu JK, Surh YJ. Nrf2-Keap1 signaling as a potential target for chemoprevention of inflammation-associated carcinogenesis. Pharm Res 2010;27:999-1013.

9. Kobayashi EH, Suzuki T, Funayama R, Nagashima T, Hayashi
$\mathrm{M}$, Sekine $\mathrm{H}$, et al. $\mathrm{Nrf2}$ suppresses macrophage inflammatory response by blocking proinflammatory cytokine transcription. Nat Commun 2016;7:11624.

10. Yang L, Shen L, Li Y, Li Y, Yu S, Wang S. Hyperoside attenuates dextran sulfate sodium-induced colitis in mice possibly via activation of the Nrf2 signalling pathway. J Inflamm (Lond) 2017; $14: 25$.

11. Lau A, Villeneuve NF, Sun Z, Wong PK, Zhang DD. Dual roles of Nrf2 in cancer. Pharmacol Res 2008;58:262-70.

12. Guo Y, Shen L. Overexpression of NRF2 is correlated with prognoses of patients with malignancies: a meta-analysis. Thorac Cancer 2017;8:558-64.

13. Torrente L, Maan G, Oumkaltoum Rezig A, Quinn J, Jackson A, Grilli A, et al. High NRF2 levels correlate with poor prognosis in colorectal cancer patients and with sensitivity to the kinase inhibitor AT9283 in vitro. Biomolecules 2020;10:1365.

14. Maines MD, Abrahamsson PA. Expression of heme oxygenase-1 (HSP32) in human prostate: normal, hyperplastic, and tumor tissue distribution. Urology 1996;47:727-33.

15. Schlager JJ, Powis G. Cytosolic NAD(P)H:(quinone-acceptor) oxidoreductase in human normal and tumor tissue: effects of cigarette smoking and alcohol. Int J Cancer 1990;45:403-9.

16. Yang $\mathrm{H}$, Wang $\mathrm{W}$, Zhang $\mathrm{Y}$, Zhao J, Lin E, Gao J, et al. The role of NF-E2-related factor 2 in predicting chemoresistance and prognosis in advanced non-small-cell lung cancer. Clin Lung Cancer 2011;12:166-71.

17. Tanaka T, Kohno H, Suzuki R, Yamada Y, Sugie S, Mori H. A novel inflammation-related mouse colon carcinogenesis model induced by azoxymethane and dextran sodium sulfate. Cancer Sci 2003;94:965-73.

18. Suzuki R, Kohno H, Sugie S, Tanaka T. Sequential observations on the occurrence of preneoplastic and neoplastic lesions in mouse colon treated with azoxymethane and dextran sodium sulfate. Cancer Sci 2004;95:721-7.

19. Thaker AI, Shaker A, Rao MS, Ciorba MA. Modeling colitisassociated cancer with azoxymethane (AOM) and dextran sulfate sodium (DSS). J Vis Exp 2012;(67):4100.

20. Tardieu D, Jaeg JP, Cadet J, Embvani E, Corpet DE, Petit C. Dextran sulfate enhances the level of an oxidative DNA damage biomarker, 8-oxo-7,8-dihydro-2'-deoxyguanosine, in rat colonic mucosa. Cancer Lett 1998;134:1-5.

21. De Robertis M, Massi E, Poeta ML, Carotti S, Morini S, Cecchetelli $L$, et al. The AOM/DSS murine model for the study of colon carcinogenesis: from pathways to diagnosis and therapy studies. J Carcinog 2011;10:9.

22. Suzuki R, Kohno H, Sugie S, Nakagama H, Tanaka T. Strain differences in the susceptibility to azoxymethane and dextran sodium sulfate-induced colon carcinogenesis in mice. Carcinogenesis 2006;27:162-9.

23. Son HJ, Sohn SH, Kim N, Lee HN, Lee SM, Nam RH, et al. Effect of estradiol in an azoxymethane/dextran sulfate sodiumtreated mouse model of colorectal cancer: implication for sex difference in colorectal cancer development. Cancer Res Treat 2019;51:632-48. 
24. Song $\mathrm{CH}$, Kim N, Nam RH, Choi SI, Son JH, Yu JE, et al. $17 \beta$-estradiol strongly inhibits azoxymethane/dextran sulfate sodium-induced colorectal cancer development in Nrf2 knockout male mice. Biochem Pharmacol 2020;182:114279.

25. Chan K, Lu R, Chang JC, Kan YW. NRF2, a member of the NFE2 family of transcription factors, is not essential for murine erythropoiesis, growth, and development. Proc Natl Acad Sci USA 1996;93:13943-8.

26. Yum HW, Zhong X, Park J, Na HK, Kim N, Lee HS, et al. Oligonol inhibits dextran sulfate sodium-induced colitis and colonic adenoma formation in mice. Antioxid Redox Signal 2013;19:102-14.

27. Cooper HS, Murthy SN, Shah RS, Sedergran DJ. Clinicopathologic study of dextran sulfate sodium experimental murine colitis. Lab Invest 1993;69:238-49.

28. Park YH, Kim N, Shim YK, Choi YJ, Nam RH, Choi YJ, et al. Adequate dextran sodium sulfate-induced colitis model in mice and effective outcome measurement method. J Cancer Prev 2015;20:260-7.

29. Choi YJ, Choi YJ, Kim N, Nam RH, Lee S, Lee HS, et al. Açaí berries inhibit colon tumorigenesis in azoxymethane/dextran sulfate sodium-treated mice. Gut Liver 2017;11:243-52.

30. Katakura K, Lee J, Rachmilewitz D, Li G, Eckmann L, Raz E. Toll-like receptor 9-induced type I IFN protects mice from experimental colitis. J Clin Invest 2005;115:695-702.

31. Parang B, Barrett CW, Williams CS. AOM/DSS model of colitisassociated cancer. Methods Mol Biol 2016;1422:297-307.

32. Boyanapalli SS, Paredes-Gonzalez X, Fuentes F, Zhang C, Guo $\mathrm{Y}$, Pung $\mathrm{D}$, et al. Nrf2 knockout attenuates the anti-inflammatory effects of phenethyl isothiocyanate and curcumin. Chem Res Toxicol 2014;27:2036-43.

33. Wu TY, Khor TO, Saw CL, Loh SC, Chen Al, Lim SS, et al. Anti-inflammatory/anti-oxidative stress activities and differential regulation of Nrf2-mediated genes by non-polar fractions of tea Chrysanthemum zawadskii and licorice Glycyrrhiza uralensis. AAPS J. 2011;13:1-13.

34. Garzón-Castaño SC, Lopera-Castrillón IA, Jiménez-González FJ, Siller-López F, Veloza LA, Sepúlveda-Arias JC. Nrf2mediated antioxidant activity of the inner bark extracts obtained from Tabebuia rosea (Bertol) DC and Tabebuia chrysantha (JACQ) G. Nicholson. F1000Res 2018;7:1937.

35. Levonen AL, Inkala M, Heikura $T$, Jauhiainen $S$, Jyrkkänen HK, Kansanen E, et al. Nrf2 gene transfer induces antioxidant enzymes and suppresses smooth muscle cell growth in vitro and reduces oxidative stress in rabbit aorta in vivo. Arterioscler Thromb Vasc Biol 2007;27:741-7.
36. Itoh K, Mochizuki M, Ishii $\mathrm{Y}$, Ishii $\mathrm{T}$, Shibata $\mathrm{T}$, Kawamoto $\mathrm{Y}$, et al. Transcription factor Nrf2 regulates inflammation by mediating the effect of 15 -deoxy- $\Delta^{12,14}$-prostaglandin $\mathrm{J}_{2}$. Mol Cell Biol 2004;24:36-45

37. Pi J, Leung L, Xue P, Wang W, Hou Y, Liu D, et al. Deficiency in the nuclear factor E2-related factor-2 transcription factor results in impaired adipogenesis and protects against diet-induced obesity. J Biol Chem 2010;285:9292-300.

38. Suzuki T, Uruno A, Yumoto A, Taguchi K, Suzuki M, Harada N, et al. Nrf2 contributes to the weight gain of mice during space travel. Commun Biol 2020;3:496.

39. Ma Q, Battelli L, Hubbs AF. Multiorgan autoimmune inflammation, enhanced lymphoproliferation, and impaired homeostasis of reactive oxygen species in mice lacking the antioxidant-activated transcription factor Nrf2. Am J Pathol 2006;168:1960-74.

40. Kim J, Cha YN, Surh YJ. A protective role of nuclear factorerythroid 2-related factor-2 (Nrf2) in inflammatory disorders. Mutat Res 2010;690:12-23.

41. Khor TO, Huang MT, Kwon KH, Chan JY, Reddy BS, Kong AN. Nrf2-deficient mice have an increased susceptibility to dextran sulfate sodium-induced colitis. Cancer Res 2006;66:11580-4.

42. Wu S, Lu H, Bai Y. Nrf2 in cancers: a double-edged sword. Cancer Med 2019;8:2252-67.

43. Myung SJ, Rerko RM, Yan M, Platzer P, Guda K, Dotson A, et al. 15-Hydroxyprostaglandin dehydrogenase is an in vivo suppressor of colon tumorigenesis. Proc Natl Acad Sci USA 2006;103:12098-102.

44. Wolf I, O'Kelly J, Rubinek T, Tong M, Nguyen A, Lin BT, et al. 15-hydroxyprostaglandin dehydrogenase is a tumor suppressor of human breast cancer. Cancer Res 2006;66:7818-23.

45. Greenhough A, Smartt HJ, Moore AE, Roberts HR, Williams AC, Paraskeva $C$, et al. The COX-2/PGE2 pathway: key roles in the hallmarks of cancer and adaptation to the tumour microenvironment. Carcinogenesis 2009;30:377-86.

46. Backlund MG, Mann JR, Holla VR, Buchanan FG, Tai HH, Musiek ES, et al. 15-Hydroxyprostaglandin dehydrogenase is down-regulated in colorectal cancer. J Biol Chem 2005;280:321723.

47. Thiel A, Ganesan A, Mrena J, Junnila S, Nykänen A, Hemmes A, et al. 15-hydroxyprostaglandin dehydrogenase is down-regulated in gastric cancer. Clin Cancer Res 2009;15:4572-80.

48. Mann JR, Backlund MG, Buchanan FG, Daikoku T, Holla VR, Rosenberg DW, et al. Repression of prostaglandin dehydrogenase by epidermal growth factor and snail increases prostaglandin E2 and promotes cancer progression. Cancer Res 2006;66:6649-56. 